\title{
DYNAMICAL PROPERTIES OF TIDALLY-INDUCED GALACTIC BARS
}

\author{
T. MIWA AND M. NOGUCHI \\ Astronomical Institute, Tohoku University \\ Aoba, Sendai 980-77, Japan
}

We have simulated a series of N-body models of tidal encounters between a disk galaxy and a perturbing galaxy to investigate the dynamical properties of tidally-induced galactic bars (Noguchi 1987, MNRAS, 228, 635). We have also calculated N-body models on isolated galaxies with bar-unstable disks (Ostriker \& Peebles 1973, ApJ, 186, 467) to make a comparison between galactic bars of different origins (Miwa \& Noguchi 1997, submitted).

It is found from our simulations that the tidally-induced bars sometimes roate quite slowly and have inner Lindblad resonances (ILR) near the bar ends, whereas the spontaneously-formed bars have no ILR and end near corotation owing to their large pattern speed. Since the difference in resonance structures affects the kinematics of the interstellar gas, these peculiar tidal bars may give us a new way of creating the vast morphological and kinematic variety observed in real barred galaxies, which may not be explained solely by the spontaneous bars.

The slow rotation of tidal bars is caused by two major factors. First, the smallness of the disk mass fraction in some models leads to a small pattern speed. Second, the angular momentum transfer from the inner disk to the perturber at perigalactic passage serves to reduce the pattern speed. "Massive disk models" in which the disk is stabilized by large random motions in the disk stars are quite sensitive to the second process.

This complicated behavior of tidal bars can be understood naturally by recognizing two regimes of tidal bar formation. When the tidal perturbation is weak, it works only as a trigger of bar formation and the bar properties are determined largely by the internal structure of the target galaxy. On the other hand, a sufficiently strong tidal perturbation washes out the intrinsic property of the target galaxy and imposes the bar as a common characteristic determined by the parameters of the tidal encounter. 